\title{
Motility Evaluation of a Novel Overground Functional Mobility Tool for Post Stroke Rehabilitation
}

\author{
J. L. Patton, Member, IEEE, D. Brown, E. Lewis, G., Crombie, , J. Santos, A. Makhlin, J. E. Colgate, \\ Member, IEEE, M. Peshkin, Member, IEEE
}

\begin{abstract}
The KineAssist is a robotic device that allows the therapist to safely interact with and challenge a patient as they train in whole body dynamic-balance tasks such as sit to stand or gait. The design and development of the KineAssist proceeded rapidly as a startup product of a small company. Here we briefly restate the KineAssist's motivation, design, and use, and then present an initial evaluation of the alphaprototype's performance on healthy and stroke survivors. The studies showed that sacral marker trajectories were slower but otherwise not significantly changed in amplitude when using the device. The KineAssist therefore yields a safe environment for training functional mobility tasks, and these results identify a promising method of safely challenging patients to exert more effort while maintaining natural kinematic ranges of motion.
\end{abstract}

\section{INTRODUCTION}

$\mathrm{R}$ ETRAINING movement skills is a critical part of recovery for the stroke surviv or, and walking and other standing dynamic balance activities are typically very high on the list of goals for the patients. Improved locomotor function can result in greater quality of life and reduction in the risk of falls. Walking speed is an effective indicator of the degree of abnormality in gait quality, ov erall functional status, and clinical progress in people with post-stroke hemiparesis $[1,2]$. Furthermore, gait speed has been found to correlate with balance and many other functional parameters [3]

However, training balance and walking often involves risk as the patient with poor balance may lose balance and fall as they learn to move while upright. While therapists try to create a safe environment, they often resort to constrained movements, and the goal of administering therapy is often distracted or overruled by concerns for safety. Our initial work presented here shows how a robotic technology can address these needs

We have previously presented a robotic design that can provide un-hindering safety and/or the ability to challenge the patient with robot-applied forces [4]. The design process began with an assessment in a variety of physical therapy

Manuscript received February 11,2007 . This work was supported in part by the U.S. Departm ent of Commerce under NIST Advanced Technology Program, Grant Award 70NANB3H3003, and by the Rehabilitation Institute of Chicago. The authors have financial interests in and/or are employed by andior are consultants to Chicago PT LLC. Several authors are affiliated with Northwestern Uriversity and/or The Rehabilitation Institute of Chicago. Contact information, affiliations, videos and more information may be found at www. chicagopt.com.

KineAssist is a registered trademark. A US patent application has been filed on the KineAssist. clinics to identify the unmet needs. Some of these unmet needs were that clinicians were unable engage in a therapeutic process while the patients were unconstrained yet safe from a fall. Another need was the ability to provide functionally relevant challenges to their patients during balance and mobility training post-stroke. Some of the other needed features were partial body weight support; the ability to provide postural torques on the torso; a safety catch as a patient begins to fall; full mobility of the legs, trunk and pelvis; full patient accessibility by a therapist, and the ability to perform motions that involve forward, rotation, and sidestepping actions.

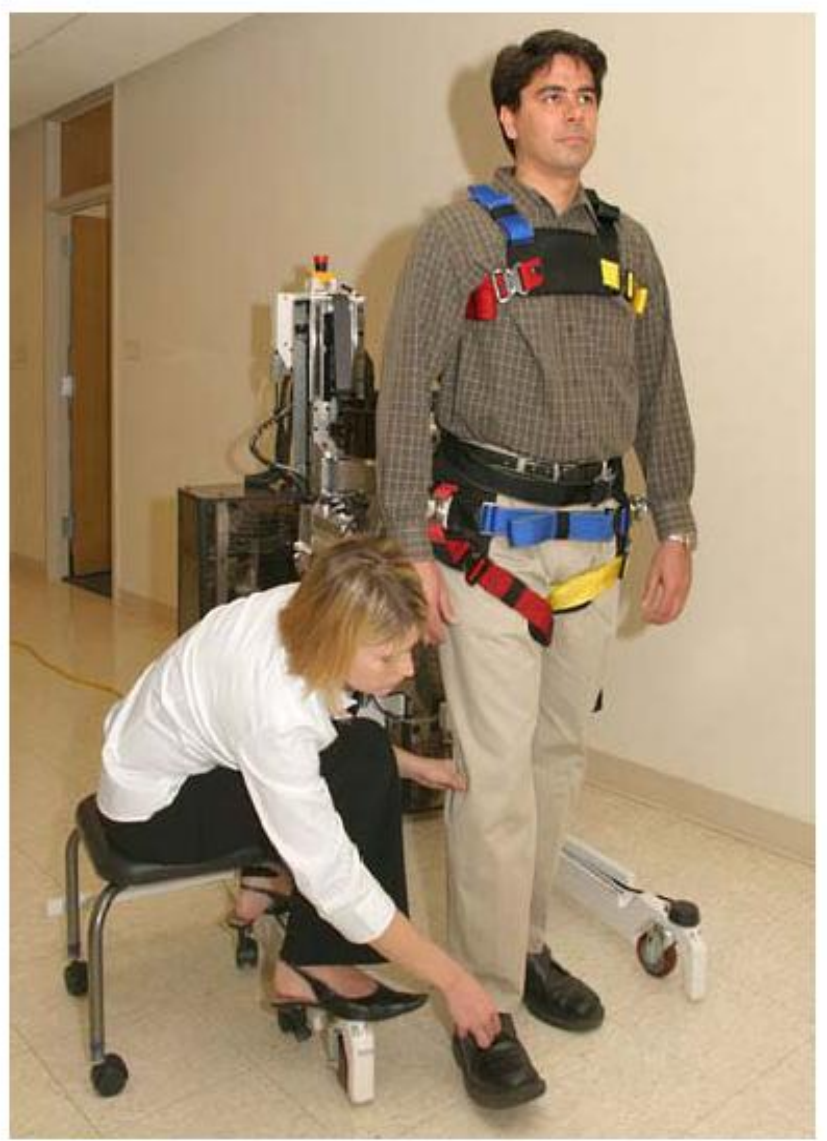

Fig 1. A therapist providing guidance during locomotion as she rides along.

The resulting KineAssist ${ }^{\mathrm{TM}}$ device servo-follows a patient's walking motions and allows clinicians to work more effectively with at-risk patients while maintaining natural movement trajectories in functional activities (Fig. 
1). The design also allows partial body weight support during mobility activities that focus on both the balance and the locomotion elements of whole body activity. Hence, one of the most preliminary questions for this device is whether it can effectively provide protection to subjects as they move without detrimentally impeding motion or corrupting the dynamic activities that the machine was meant to help improve.

We present two important initial studies on the KineAssist alpha-1-prototype's performance for four common standing dynamic activities: walking, sit-to-stand, stand-to-sit, and reaching for an object. The first experiment evaluates the transparency of the device on healthy subjects by evaluating their activity inside and outside of the device. The second experiment evaluates whether the device is effective for training stroke survivors. These experiments showed two main results. First, the sacral trajectories in all planes inside the device were slowed down and the amplitude was dampened but not changed from the same task performance outside of the device, therefore, yielding a safer environment for training functional mobility tasks. Secondly, EMG activity was heightened, indicating that such practice on tasks using the same kinematics require more effort. These results identify a promising method of safely challenging patients to exert more effort while maintaining natural kinematic ranges of motion.

\section{DESCRIPTION OF THE KinEAssist DEsign}

The two major components are a mobile base system and a brace system. The two systems are further broken down into subsystems described below. The mobile base is omnidirectional and uses Cobot technology originally developed by Peshkin and Colgate for assistive devices in materials handling [5]. This admittance control methodology renders a haptic display that compensates for the inertial effects of the robot, rendering the system virtually undetectable and allowing easy forward and turning motions while the machine moves in response to the motion of the patient. Force sensed at the pelvic harness is used to drive the motion of the system. The trunk and pelvis mechanism allows the patient's bending motions both left/right, forward/backwards, rotations about a person's transverse axis, and hip rotations about the forward axis. A torso mechanism attaches at chest level and can prevent collapse of the trunk. A software-driven "Safety Zone" limits the patient's upper body range of motion where the trunk support implements an adjustable, compliant constraint that catches the patients when they lose balance. In addition to simply acting as a fall-arresting device, the patient's weight can be partially supported at the level of the pelvis, and the system is also capable of comfortably applying forces to the body (although these features were not used in the experiment presented below). The therapist has the freedom to change parameters and assist or challenge the patient to the level they determine is necessary to gain the best clinical outcomes.
We have discovered several significant challenges in this design cycle. One challenge was to design an appropriate pelvis interface that accommodates a wide variety of body shapes while allowing the necessary degrees of freedom and control from the robot. Another challenge was to instantaneously move a heavy (approx 400 pounds) robotic mechanism, designed firstly for safety, in response to very small motions and forces exerted by the patient. Adaptive haptic algorithms improved this transparency aspect of the device. The controls also had to accommodate specific modes of operation, because an impending fall is defined differently for walking than for sit-to-stand.

\section{EXPERIMENTAL PROCEDURES}

We performed validation of the transparency of the system during common training tasks in the clinic. The study compared the difference in sacral motion and EMG muscle activity of common activities performed in neurological rehabilitation both inside the device and outside the device. We also compared the difference between individuals without neurological impairments (Experiment 1) to individuals with post-stroke hemiplegia (Experiment 2). Each experiment's methods are detailed below.

\section{A. Experiment 1: Transparency on healthy subjects}

Five healthy individuals ( 4 Female, ages ranging from 24 to 31) with no known neuromuscular disorders participated in the experiment after giving informed consent in accordance with the Institutional Review Board standards of Northwestern University. Our goal was to address how the KineAssist device may alter the motion of healthy individuals, while performing four functional tasks:

1) Sit to Stand. Participants were asked to sit on a bench without back support and instructed to sit as safely as possible, not using arms and remain standing and looking forward at the end of the trial.

2) Stand to sit. Participants were asked to stand in front of the bench and instructed to sit down as safely as possible, without using arms and remain sitting, looking forward at all times.

3) Forward reach. Subjects reached forward while standing. A piece of paper was taped to a $0.254 \mathrm{~m}$ tall block, which was placed in front of the subject's feet. Participants were asked to reach forward, touch the piece of paper and then return to the starting standing position.

4) Walking forward. Participants were asked to walk over a 15 meter walkway and then stop and remain facing forward.

All subjects completed a series of these four tasks, first while out of the KineAssist and then while attached to the KineAssist. Prior to the beginning of each task, subjects were allowed time to practice each task in the device, to ensure comfort and safety of the test. Three speeds were completed and described to the subject as slow, comfortable and fast. Subjects completed three trials in each condition in 
order to assess reliability.

All four functional tasks were performed on a 15 meter walkway using advanced hardware and software for real time reconstruction of body-segment motions using the spherical markers. Motion was tracked using a Motion Analysis System (Motion Analysis Corp., Santa Rosa, CA) that uses eight charge-coupled video cameras (VC491; Oxford) in an optimal redundant configuration. Seven spherical markers, $0.02554 \mathrm{~m}$ in diameter where placed on the sacrum, and left and right upper and lower thighs. To determine a baseline anatomical frame of reference, additional markers were placed at left and right Anterior Superior Iliac Spine (ASIS) and were removed prior to performing the tasks. Measurement data were collected and post-processed using EVART software (Motion Analysis corporation, CA), which is equipped to calculate body's center of mass trajectories using anatomical models of mass distribution and segmental geometry. Four footswitches were placed on each foot to record floor contact events. Force plate data was available but not used.

We collected six EMG signals on each lower extremity (Motion Lab Systems, Inc., Baton Rouge, LA USA) : rectus femoris, vastus medialis, biceps femoris, semimembranosis, medial gastrocnemious and anterior tibialis. EMG was rectified and summed for each muscle. Our analysis focused on understanding the total difference in effort between performances in and performances out of the KineAssist. Each summed muscle activity pattern was then normalized to the summed amount of that muscle when performing the task without the KineAssist. This allowed a percentage change calculation.

TABLE I

\begin{tabular}{|c|c|c|c|c|c|c|c|}
\hline \multicolumn{8}{|c|}{$\begin{array}{c}\text { TABLE } 1 \\
\text { STROKE SURVIVOR PROPERTIES }\end{array}$} \\
\hline & 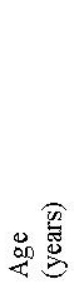 & 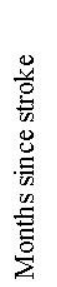 & 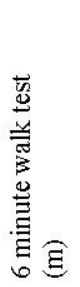 & 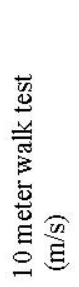 & 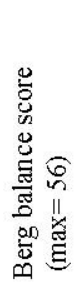 & 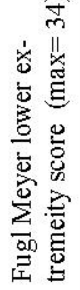 & 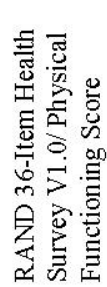 \\
\hline Mean & 60.4 & 129 & 266.9 & 1.21 & 52.1 & 21.5 & 73.83 \\
\hline St. Dev. & 10.8 & 81 & 87.42 & 0.29 & 3.1 & 6.08 & 16.35 \\
\hline
\end{tabular}

\section{B. Experiment 2: KineAssist Efficacy with stroke survivors}

Ten volunteers who were greater than six months poststroke participated in this study; 4 were female, 3 were right side paralyzed, 4 regularly ambulated using an orthoses and 4 regularly ambulated using a standard cane. This experiment differed from the first experiment in only two ways. First, after giving informed consent, a physical therapist first characterized the functional limitations of each stroke participant using four clinical measures: 1) 10-Meter Walk Test [6], 2) Six-Minute Walk Test [7, 8], 3) Rand 36Item Health Survey Version 1.0 Physical Functioning Score [9] and 4) Berg Balance Test [10], 5) Fugl-Meyer [11].
Demographics of each subject and the results of these tests are shown in Table 1. The second difference from the first experiment is that subjects were only asked to complete three trials of each task at a single (comfortable) speed.

\section{RESULTS}

The clinical study results were twofold and were similar for both healthy and stroke groups. First, the KineAssist did not alter the kinematics of several functional activities. These activities included sit to stand, stand to sit, forward reaching and walking. In addition, the study showed that the subjects worked harder via EMG recordings of six lower extremity muscle groups.

We investigated the range of motion in this preliminary study by focusing on the sacral marker on each subject, which closely approximates the motion of the center of mass. We found surprisingly small differences in the overall kinematic ranges of motion for the sacrum (Figures $2-4)$. For the healthy group, the only significant reduction in range of motion was observed at the fast speed and only for the vertical range of motion (Fig $4 \mathrm{~A}$ ). Hence kinematic motions were possible and only slightly reduced. In experiment 2 on individuals with a history of stoke, we observed a reduction in the vertical range of motion of the sacrum at the comfortable (self-selected) speed.

In contrast to relatively unchanged ranges of motion, both healthy and stroke survivors' peak velocities were smaller in the KineAssist (bottom bar charts of Fig. $4 \mathrm{~A}$ and B). Differences in vertical velocity were detectable at faster speeds.

Finally, the EMG data showed that there was a significant increase in muscle activity when the subjects used the KineAssist device (Fig 5). This increase was evident for both healthy and stroke groups, in both paretic and nonparetic limbs, for all tasks. Consequently, the KineAssist requires more apparent neural drive or effort from its user.

\section{DISCUSSION}

A novel approach in rehabilitation robotic design was utilized by starting with the end user and implementing the feedback received to create a device that assists with functional mobility in stroke rehabilitation. The KineAssist device can work with a therapist during a multitude of functional tasks that typically take place during a neurological physical therapy session. The design objectives for mechanical and control systems were unique in that the KineAssist should allow relatively "transparent" movement during functional mobility tasks. The focus of this paper was to evaluate this objective, and to determine just what happens to kinematics and EMG when a person (either healthy or a person with a history of stroke) goes from performing a functional task without the machine to performing inside the machine. While the device is not transparent, it appears that the effect of the KineAssist device does not significantly alter the range of motion in individuals. The device simply slows them down and 
requires slightly more effort.

Even though overall the device performed well, we did observe some limitations late in the design evaluation. First and foremost, a slower response time in the functional mobility activities was programmed so individuals with poor motor control would not be able to lose control of the device. Secondly, a large load in the back of the device was necessary so that a cantilever effect would not make the device unstable when a patient places a large portion of weight on the pelvic harness (such as when the device catches people from falling).

An "alpha-two" prototype is currently being built which will address several of these limitations. It will also be altered to allow free use by clinicians with little or no training. This will be part of further clinical testing and will validate several the software components that make the device unique. These include a more effective body weight support overground; perturbations in several functional environments, activities that involve a large range of motion such as transitions from the floor, and the software that allows several new activities that can include induced imbalance.

In conclusion, a novel approach was taken in the concept, design and development of a robotic device in physical therapy. The project has resulted in a device that will work with physical therapists to help them perform interventions more efficiently and effectively in the area of overground gait and balance training. Therapists that were once limited in their ability to challenge their patients by either a) the limits of their own strength or b) the availability of other staff members to help them train their patients will no longer have to deal with these training boundaries when planning and implementing clinical intervention. Ultimately, this will allow individuals affected by a stroke and other neurological impairments to safely be challenged to their physical limit versus the physical limits of the individual therapist. This preliminary study has shown that the KineAssist can challenge a patient without impeding natural kinematics.

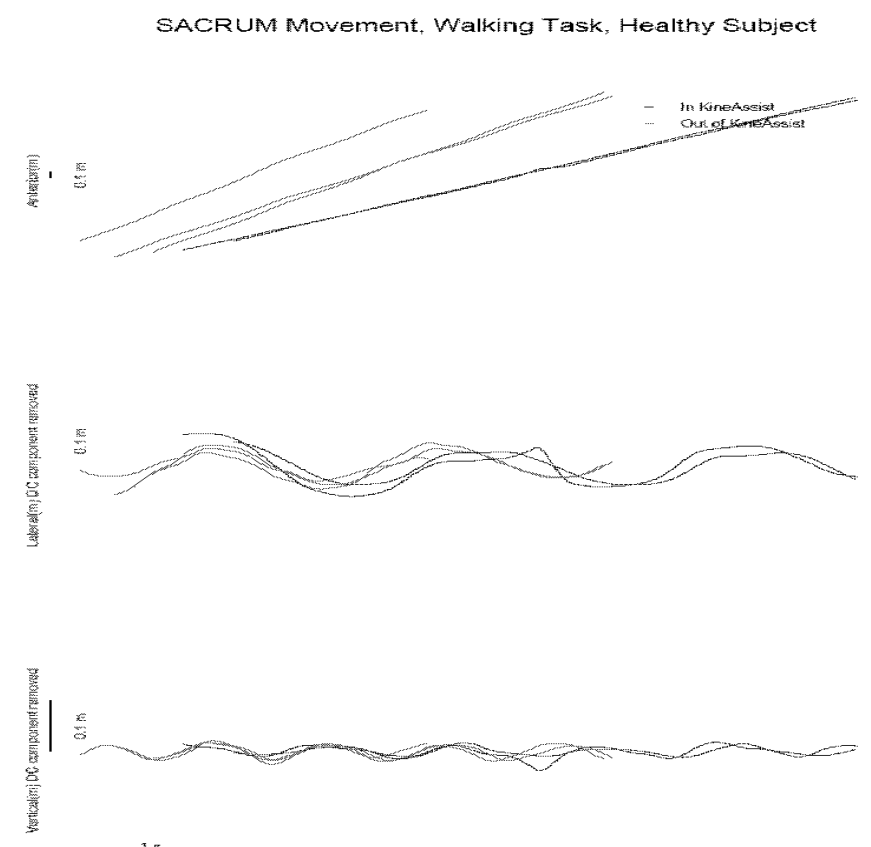

Fig. 2. Typical kinematic trajectories of the sacrum marker for a healthy subject walking in and out of the KineAssist device. Here three trials were collected for each task. The left foot strike was used to align all data in time. The slopes of the data reveal a slightly slower speed but otherwise little or no change in the range of motion. Note that due to limitations in what the makers could be seen by the motion capture system, the recorded span time varies for each trial.

SACRLM Movement, Waking (Self-Selected Speed), Stroke Subject
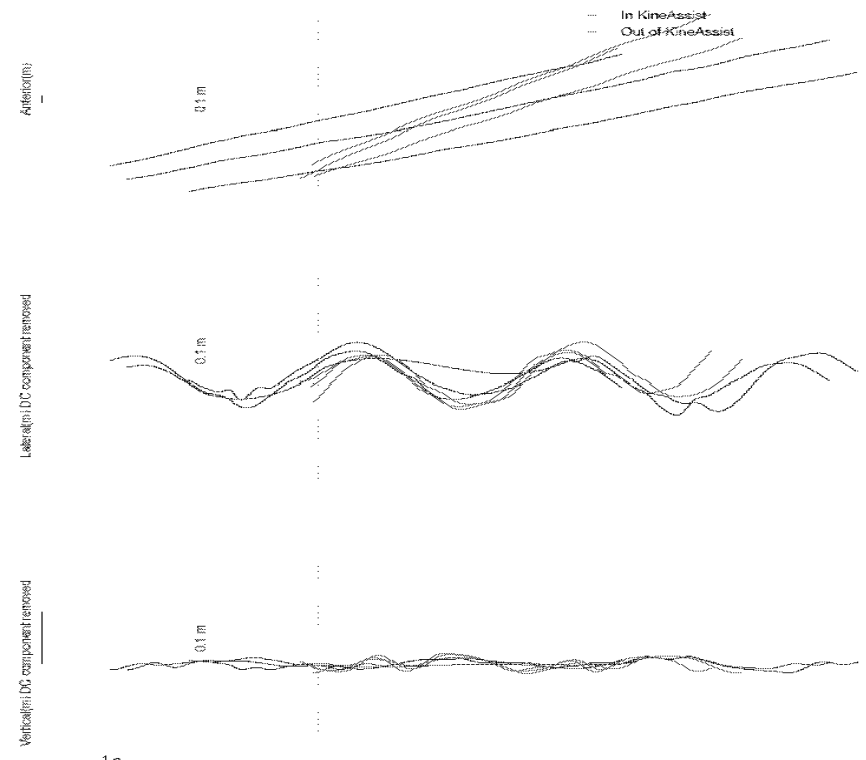

Fig. 3. Typical kinematic trajectories for an individual with a history of stroke, in and out of the Kine Assist device. This figure uses the same conventions that were used in Fig. 2. 


\section{A. HEATHY:}

SLOW SPEED

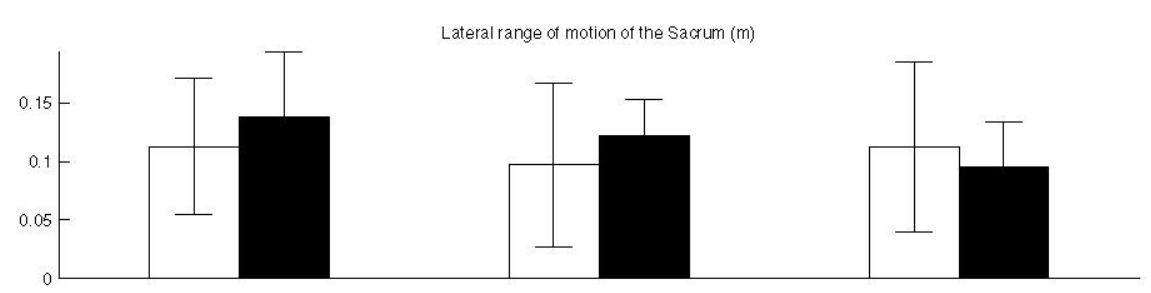

Vertical range of motion of the Sacrum (m)
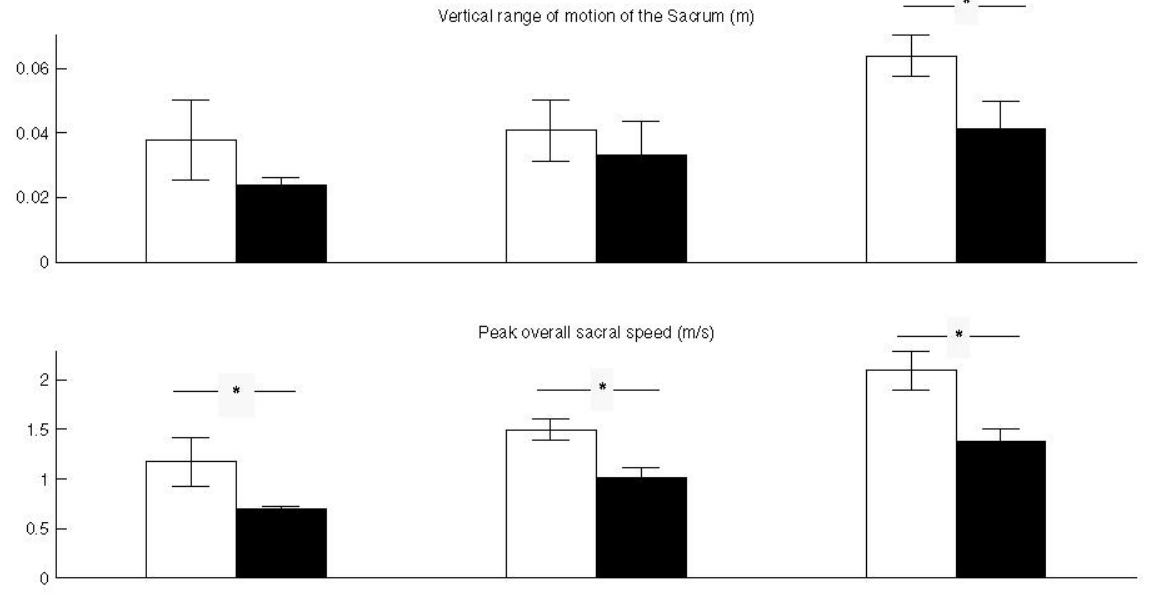

Peak lateral sacral veloccty $(\mathrm{m} / \mathrm{s})$

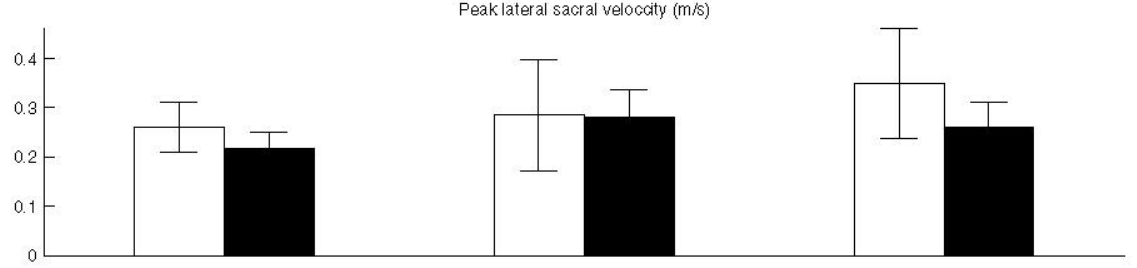

Peak veritcal sacral velocity $(\mathrm{m} / \mathrm{s})$

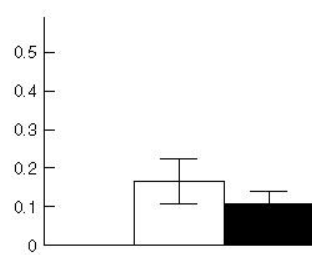

\section{B. STROKE:}

COMFORTABLE SPEED

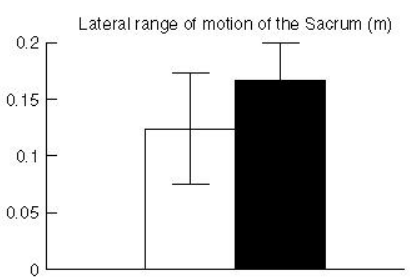

Vertical range of motion of the Sacrum (m)

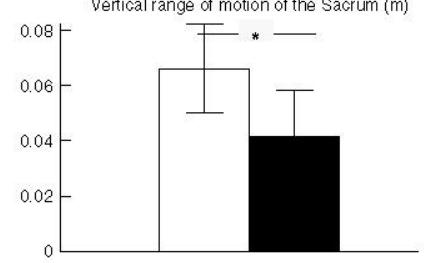

Peak overall saral speed $(\mathrm{m} / \mathrm{s})$

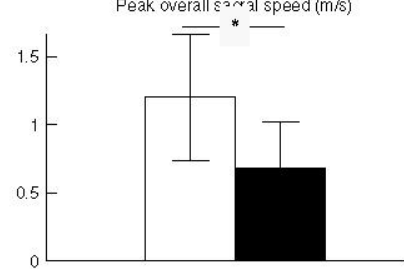

Peak lateral sacral velocity (m/s)
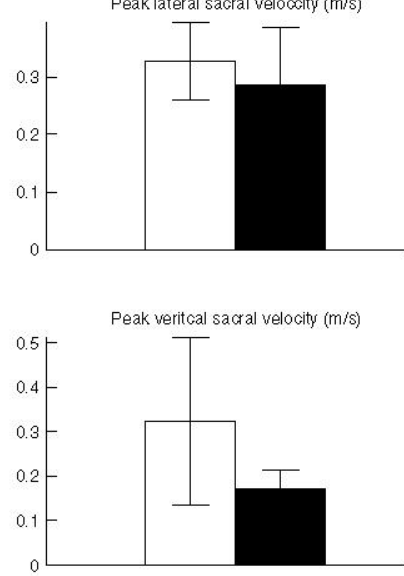

Fig. 4. Group kinematic measures for the Healthy Group for walking in the KineAssist. For the Healthy (A) and Stroke survivor (B) groups. Healthy subjects completed three speeds of walking while stroke survivors completed walking at a comfortable speed. White bars show values taken while subjects were inside the KineAssist, while black bars show values out of the KineAssist. Statistics for all subjects were compiled from averages of all trials in each condition. Wings show $95 \%$ confidence intervals. Significant difference based on a paired t-test $(\alpha=0.05)$ is indicated by an asterisk and horizontal line above each plot. 


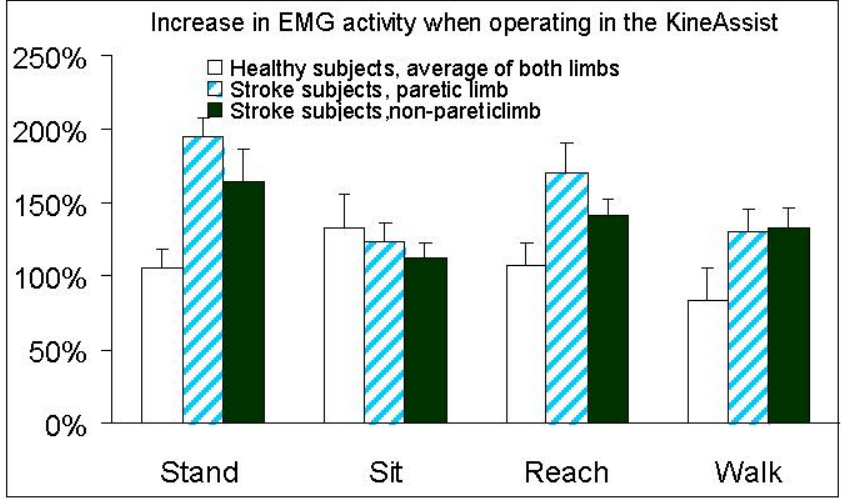

Fig. 5. Increase in the EMG when using the KineAssist. Each muscle EMG was rectified and summed for the task. Each muscle was then normalized to its amount when performing the task outside the KineAssist. Shown here is the increase in all muscles when subjects performed the tasks using the KineAssist, as a percentage of activity outside the KineAssist. For example, a 100\% increase means that the summed EMG when using the KineAssist was twice that of the EMG when operating without the KineAssist. Wings show standard Error

\section{ACKNOWLEDGMENT}

We wish to thank Rehabilitation Institute of Chicago and the U.S. Department of Commerce NIST Advanced Technology Program for support of this work. Amy Schwartz and Ben Rush of IDEO-Chicago skillfully guided our user needs analysis and choice of technical target. We appreciate the contributions of Tom Moyer and Doug Schwandt for their help on the mechanical design of the KineAssist. We thank the many clinicians, administrators, and patients who repeatedly shared their perspectives, experiences, advice, and expertise with us.

\section{REFERENCES}

[1] M. Dettmann, M. Linder, and S. Sepic, "Relationships among walking performance, postural stability, and functional assessments of the hemiplegic patient," Am J Phys Med Rehabil, vol. 66, pp. $77-90,1987$.

[2] E. Knutsson, A. Martensson, and L. Gransberg, "Influences of muscle stretch reflexes on voluntary, velocity-controlled movements in spastic paraparesis," Brain, vol. 120, pp. 1621$1633,1997$.

[3] E. Roth, C. Merbitz, and K. Mroczek, "Hemiplegic gait: relationships between walking speed and other temporal parameters," Am J Phys Med Rehabil vol. 76, 1997.

[4] M. Peshkin, D. A. Brown, J. J. Santos-Munné, A. Makhlin, E. Lewis, J. E. Colgate, J. L. Patton, and D. Schwandt, "KineAssist: A robotic overground gait and balance training device," presented at IEEE-International Conference on Rehabilitation Robotics (ICORR), Chicago, IL, USA, 2005.

[5] J. E. Colgate, W. Wannasuphoprasit, and M. A. Peshkin, "Cobots: Robots for Collaboration with Human Operators," Proceedings of the International Mechanical Engineering Congress and Exhibition, Atlanta, GA, vol. 58, pp. 433-439, 1996.

[6] N. M. Salbach, N. E. Mayo, J. Higgins, S. Ahmed, L. E. Finch, and C. L. Richards, "Responsiveness and predictability of gait speed and other disability measures in acute stroke," Archives of Physical Medicine \& Rehabilitation, vol. 82, pp. 1204-12, 2001.

[7] A. T. S. AmericanThoracicSociety and C. o. P. S. f. C. P. F. Laboratories, "ATS statement: guidelines for the six-minute walk test.[see comment]," American Journal of Respiratory \& Critical Care Medicine, vol. 166, pp. 111-7, 2002.

[8] P. S. Pohl, P. W. Duncan, S. Perera, W. Liu, S. M. Lai, S. Studenski, and J. Long, "Influence of stroke-related impairments on performance in 6-minute walk test," Journal of Rehabilitation Research and Development, vol. 39 pp. 439-444, 2002.

[9] R. Hays, C. Sherbourne, and R. Mazel, "The RAND 36-Item Health Survey 1.0," Health Economics, vol. 2, pp. 217-227, 1993.

[10] H. F. Mao, I. P. Hsueh, P. F. Tang, C. F. Sheu, and C. L. Hsieh, "Analysis and comparison of the psychometric properties of three balance measures for stroke patients," Stroke, vol. 33, pp. 1022-7, 2002.

[11] A. R. Fugl-Meyer, L. Jaasko, I. Leyman, S. Olsson, and S. Steglind, "The post-stroke hemiplegic patient. 1. a method for evaluation of physical performance," Scandinavian Journal of Rehabilitation Medicine, vol. 7, pp. 13-31, 1975. 